\title{
Detection of Copy Number of Mitochondrial DNA in Iraq Population
}

\author{
Suhair M. Jabbar' ${ }^{1}$, Nihad A.M. Al-Rashedi \\ ${ }^{1}$ Post Graduate, ${ }^{2}$ Prof., Department of Biology, College of Science, Al Muthanna University, Samawah, Iraq
}

\begin{abstract}
Mitochondrial DNA copy number (mtDNA-CN), a measure of the number of mitochondrial genomes per cell. A cell contains two copies of genomic DNA compared to a single copy of DNA per chromosome. The quantitative real-time PCR (qPCR) is the gold standard method for measuring mtDNA-CN, the quantification after DNA extraction it is an important step, the quantitation of DNA plays a central role inmedical and forensic DNA analysis, which provides the information about the amount of DNA present in unknown samples. In this study the quantitative results showed that the higher value 74941 and the lower value 190 . A multiplex quantitative PCR assay developed to amplify target sequences of different length, which allows for the assessment of DNA degradation in samples of forensic interest. The quantification of DNA by qPCR relies on the detection of amplified product (amplicon) at each cycle of the PCR.
\end{abstract}

Keywords: Mitochondrial DNA, copy number, qPCR,Iraq population

\section{Introduction}

Mitochondrial DNA copy number (mtDNA-CN) is increasingly used to assess the role of mitochondria in forensic field and is applicable when samples that fail to yield successful nuclear DNA profiles, in cases where nuclear DNA is significantly degraded, cannot be isolated or is present in extremely limited amounts. ${ }^{[1,2]}$.

Quantitative real-time PCR (qPCR) has been the most widely used method for measuring mtDNA-CN, partly due to its low cost and quick turnaround time. However, these advances, it is important for the field to evaluate these method in the context of the current gold standard.

In addition to the method for determining mtDNA$\mathrm{CN}$, it is important to consider the impact of DNA extraction method on mtDNA-CN, particularly due to the small size and circular nature of the mitochondrial

\section{Corresponding Author:}

\section{Suhair M. Jabbar}

Post Graduate, Department of Biology, College of

Science, Al Muthanna University, Samawah, Iraq

e-mail: sohaiermahdi@yahoo.com genome. Previous research has shown organic solvent extraction is more accurate than silica-based method at measuring mtDNA-CN, which is unsurprising as column kit parameters are typically optimized for DNA fragments $\geq 50 \mathrm{~Kb}^{[3]}$. However, as all DNA extraction method have bias in the DNA which they target, measuring mtDNA-CN from direct cell lysate may prove to be a more accurate method.

\section{Method and Materials}

The study recruited 100 individuals from different south regions of Iraq between 18 and 65 years. In workflow of experiment, DNA isolated from whole blood, the copy number of mitochondrial DNA (mtDNA) was estimated by QPCR analysis using the mitochondrial gene NADH dehydrogenase subunit 1 ND1 and ND5. ND1 and ND5 copy number were normalized to half the level of GAPDH since each cell contains two copies of genomic DNA compared to a single copy of DNA per chromosome. Each sample was run in triplicate, and QPCR analysis was performed.

The qPCR reactions were performed by using the SYBR- Green qPCR, Real-time PCR analysis of two mtDNA targets and one nuDNA targets relative quantification of the copy number of mtDNA using 
calculate the copy number of mtDNA from the $\mathrm{Ct}$ values obtained for each of the three target genes.

\section{Calculation:}

a. Determination of the difference in the $\mathrm{Ct}$ values for the ND1/GAPDH pair

$$
\begin{aligned}
& (\Delta \mathrm{Ct} 1=\mathrm{Ct} \text { for GAPDH }-\mathrm{Ct} \text { for ND1 }) \\
& \Delta \mathrm{Ct} 1=\mathrm{Ct} \text { GAPDH }-\mathrm{CtND} 1
\end{aligned}
$$

b. Determination ofthe difference in the $\mathrm{Ct}$ values for the ND5/GAPDH pair in thesame manner.

$$
\Delta \mathrm{Ct} 2=\mathrm{Ct} \text { GAPDH }-\mathrm{Ct} \text { ND5 }
$$

c. To Find $2^{\Delta \mathrm{Ct}}$ for the values for $\Delta \mathrm{Ct} 1$ and $\Delta \mathrm{Ct} 2$.

Relative mitochondrial DNA content $=2 \times 2^{\Delta \mathrm{CT}}$

d. Use the average of the 2 values found in step c. as mtDNA copy number ${ }^{[4]}$.

\section{Results and Discussion}

The DNA yield of extraction was subject to estimated copy number of mitochondrial DNA (mtDNA) to nuclear DNA (nuDNA) ratio for each sample. The qPCR analysis was by using the mitochondrial gene NADH dehydrogenase subunit 1 (ND1) and ND5, the nuclear gene Glyceraldehyde 3-phosphate dehydrogenase (GAPDH). In this study the differences of $\mathrm{Ct}$ values both mtDNA and nuDNA Ct values was measured from realtime PCR, and average the CT values from triplicate reactions mtDNA copy number to relative nuDNA amounts were calculated the copy number by using the following equations:

$$
\Delta \mathrm{CT}=(\text { nuDNA CT }- \text { mtDNA CT })
$$

Relative mitochondrial DNA content $=2 \times 2 \Delta \mathrm{CT}$

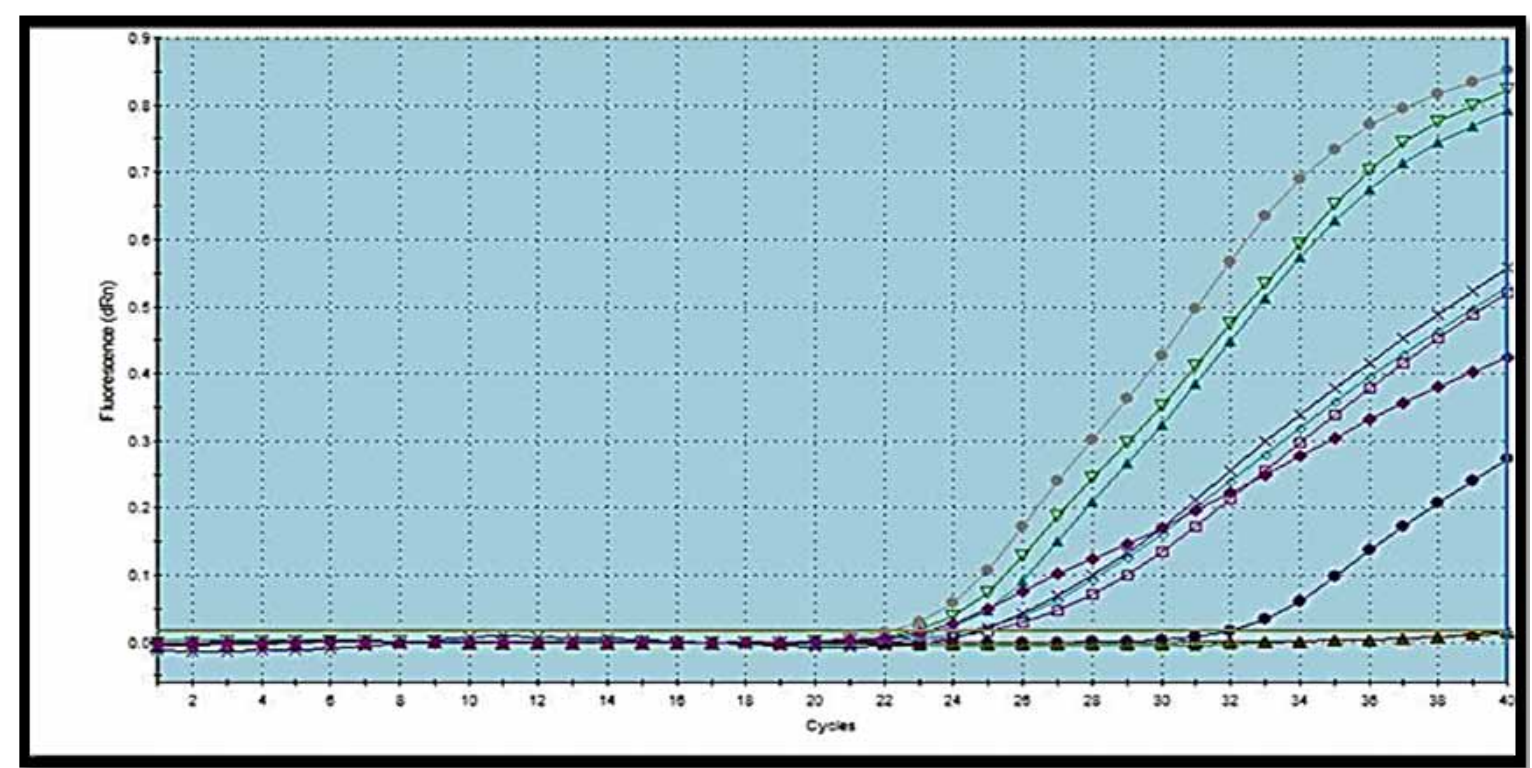

Figure (1): SYBER -Green amplification plot of ND1, ND5, and GAPDH genes for detection of nuclear DNA to mtDNA ratio.

The design of the mitochondrial qPCR assay selected a region of the ND1(NADH dehydrogenase subunit 1) gene corresponding to bases 3485-3553 of the Cambridge Reference Sequence (CRS) in order to improve the accuracy and precision of DNA quantifications ${ }^{[5]}$. The reason for chosen this target was previous cross-species sequence homology studies to indicated this sub region of the ND1 gene to be significantly non conserved a promising feature for developing a species specific assay and because this region of ND1 has few known SNPs especially when compared to the HV1,HV2 in control regions using a number of web based searching tools and avoiding any well-known disease associated SNPs such as the LHON associated SNP at CRS3460 designed and evaluated several assays at the ND1 target ${ }^{[6]}$. 


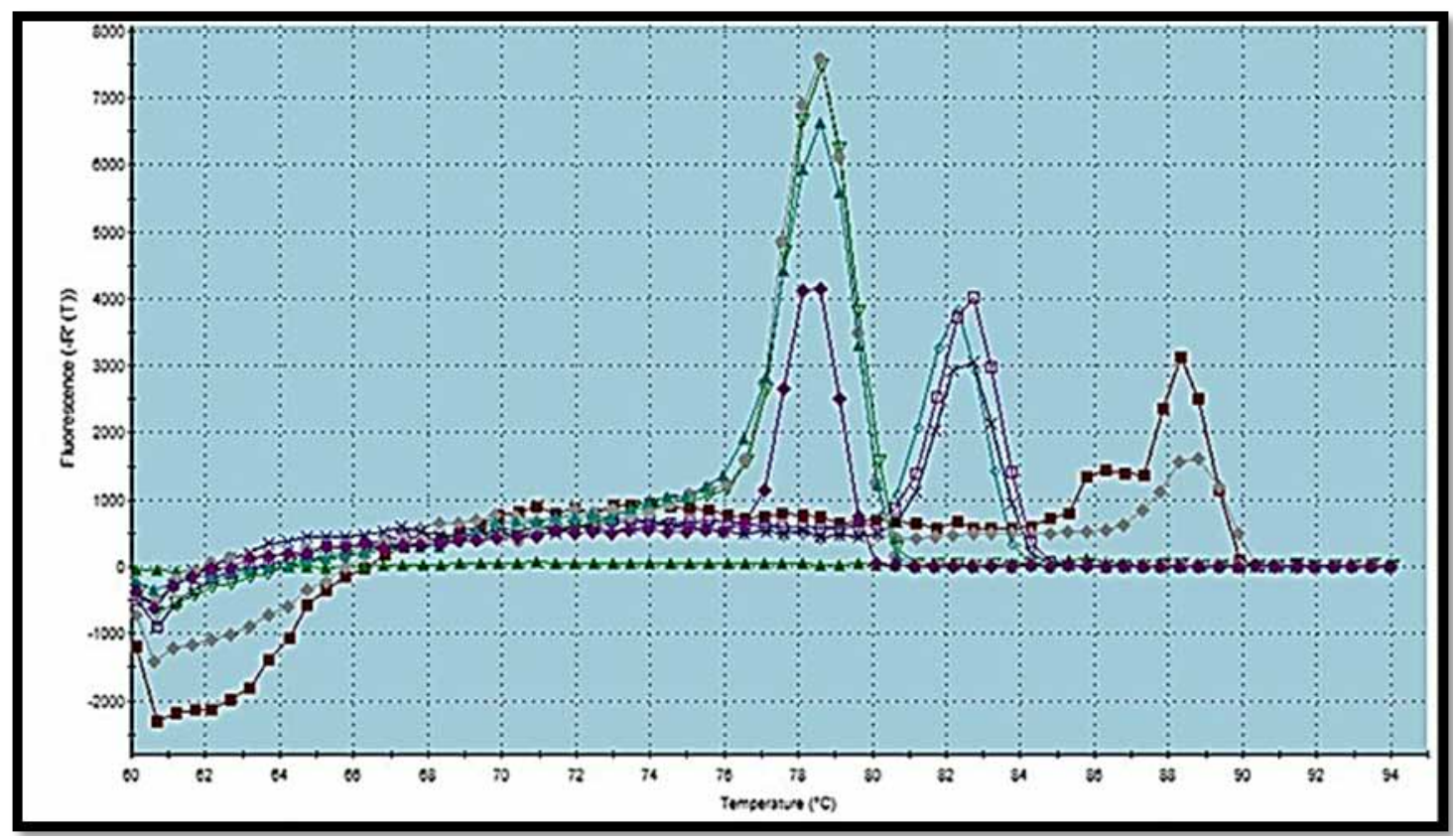

Figure (2): SYBER -Green melting curve of ND1, ND5, and GAPDH genes for detection of nuclear DNA to mtDNA ratio.

In order to process a successful duplex qPCR assay it is necessary to identify reaction conditions that effectively allow two amplifications to occur independently in the same tube the goal is to avoid the predicament in which one of the amplifications reduces the PCR efficiency of the second amplification such as situation can lead to a delayed amplification for the second assay an artificially large CT value and a corresponding underestimation of the quantity of target DNA measured by that assay one strategy for avoiding this situation is to develop duplexed qPCR assay to run under conditions that limit PCR amplification of the more abundant target sequence for example by limiting the primer concentrations for this amplification ${ }^{[7]}$.

Ethical Clearance: The Research Ethical Committee at scientific research by ethical approval of both environmental and health and higher education and scientific research ministries in Iraq

Conflict of Interest: The authors declare that they have no conflict of interest.

Funding: Self-funding

\section{Reference}

1. B. Budowle, M.W. Allard, M.R. Wilson, R. Chakraborty, Forensics and mitochondrial DNA: applications, debates, and foundations, Annu. Rev.
Genomics Hum. Genet. 4 (2003) 119-141.

2. P. Gill, P.L. Ivanov, C. Kimpton, R. Piercy, N. Benson, G. Tully, I. Evett, E. Hagelberg, K. Sullivan, Identification of the remains of the Romanov family by DNA analysis, Nat. Genet. (1994); $6: 130-135$.

3. Guo W, Jiang L, Bhasin S, Khan SM, Swerdlow RH. DNA Extraction Procedures Meaningfully Influence qPCR-Based mtDNA Copy Number Determination. Mitochondrion. 2009; 9(4):261265.

4. Furda, A. M; Bess, A.S; Meyer, J. N; Van Houten, B. Analysis of DNA damage and repair in nuclear and mitochondrial DNA of animal cells using quantitative PCR. Method Mol. Biol. (2012); 920:111-132.

5. Anderson, S; Bankier, A. T; Barrell, B. G; de Bruijn, M.H; Coulson, A. R; Drouin. Sequence and organization of the human mitochondrial genome. Nature, (1981); 290: 457-465.

6. Cummings, Michael P., Sarah P. Otto, and John Wakeley. "Sampling properties of DNA sequence data in phylogenetic analysis." Molecular Biology and Evolution (1995); 12.5: 814-822.'

7. Fixman, M; and Freire, J.J.Theory of DNA melting curves in phylogenetic analysis. Mol. Biol. Evol. (1995).12:814-22. 